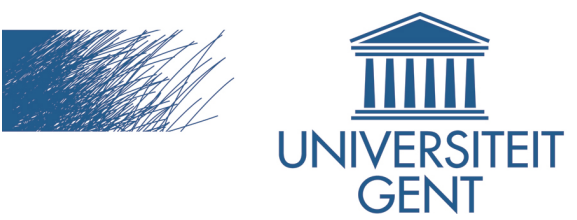

biblio.ugent.be

The UGent Institutional Repository is the electronic archiving and dissemination platform for all UGent research publications. Ghent University has implemented a mandate stipulating that all academic publications of UGent researchers should be deposited and archived in this repository. Except for items where current copyright restrictions apply, these papers are available in Open Access.

This item is the archived peer-reviewed author-version of:

Minimum commuting distance as a spatial characteristic in a non-monocentric urban system: The case of Flanders

Boussauw, K., Neutens, T. \& Witlox, F.

In: Papers in Regional Science (forthcoming, doi:10.1111/j.1435-

5957.2010.00295.x).

To refer to or to cite this work, please use the citation to the published version:

Boussauw, K., Neutens, T. \& Witlox, F. (2010). Minimum commuting distance as a spatial characteristic in a non-monocentric urban system: The case of Flanders. Papers in Regional Science (forthcoming). doi:10.1111/j.14355957.2010.00295.x 


\title{
Minimum commuting distance as a spatial characteristic in a non- monocentric urban system: The case of Flanders*
}

\author{
Kobe Boussauw ${ }^{1}$, Tijs Neutens ${ }^{1}$, Frank Witlox ${ }^{1}$ \\ 1 Geography Department, Ghent University, Krijgslaan 281 S8, B-9000 Gent, Belgium \\ (e-mail: kobe.boussauw@ugent.be,tijs.neutens@ugent.be, frank.witlox@ugent.be)
}

\begin{abstract}
This paper focuses on regional variations in commuting trip lengths by calculating minimum (required) commuting distances, along with excess commuting rates. The study contributes to the excess commuting research framework from a regional perspective, both by stressing the specific characteristics of urban networks with overlapping commute areas, and by putting forward an alternative method for calculating spatially disaggregated values. A case study in the north of Belgium shows that large variations in minimum commuting distances occur. This in turn identifies to a large extent opportunities for shrinking commuting distances by influences such as rising fuel prices, compact urban planning, extreme congestion or dissuasive traffic policies.
\end{abstract}

JEL classification: R14, R23, R40

Key words: Excess commuting, spatial proximity, sustainable spatial development, Flanders

\section{Introduction}

The concept of excess commuting or wasteful commuting was initially introduced by Hamilton (1982), and has become a well-established line of inquiry in transportation research

\footnotetext{
* This research has been made possible within the Flemish Policy Research Centre for Housing and Space, funded by the Ministry of the Flemish Community. The authors wish to express their thanks to the Flemish Traffic Control Centre for the assistance. Also, we would like to thank the two anonymous referees for their useful comments which led to significant improvements of the paper. All remaining errors are ours.
} 
in the last decades (Ma and Banister 2006). Hamilton (1982, p. 1040) defined wasteful commuting as the difference between the actual commuting distances and the theoretical minimum (required) commuting distances, which are suggested by the spatial structure of a considered city. Hamilton's interest for minimized commuting distances probably stemmed from the consecutive oil crises of 1973 and 1979-1980, when the availability, and in particular, the affordability of fossil oil products was at stake. Daily trips over large distances were suddenly considered problematic, because of their particularly high energy consumption. There is a broad consensus that spatial structure as a combination of morphological elements and activities (e.g. size, shape and functional mix) is a key determinant in explaining travel pattern generation (Giuliano and Small 1993; Van Acker et al. 2007). In many policy documents on mobility and transport much hope is set on achieving an 'adequate spatial planning' as an effective means to improve the efficiency and sustainability of mobility. However, while the spatial structure is generally recognized as a prerequisite for trip generation, observed travel behaviour and trip distances in particular are additionally induced by other factors.

We know that the observed average commuting distances in European and North American metropolitan areas increase year after year (Banister et al. 1997; Aguilera 2005). There is no doubt that the increased prosperity implicitly or explicitly plays a role in the growth of the travelled distances. However, it appears that the possibility to travel over increasingly larger distances is systematically materialized in the form of the physical separation of functions. Given that the public debate acknowledges that an important part of the traffic problems is related to land use policies, it is important to estimate what share of the actual travel is caused by the spatial structure itself and what share is in fact an extrapolation that originated from other elements, such as the general prosperity, but also the level of congestion, the quality of roads, or the price of fuel. Hamilton (1982) explored only home-work commuting, but the 
concept may, mutatis mutandis, be extended to all daily travel categories (Horner and O'Kelly 2007).

Researchers in this field usually consider excess commuting as a characteristic of a specific city, regarded as a monocentric, polycentric or dispersed urban system. Building on the exploratory work by Niedzielski (2006) and Yang and Ferreira (2008) we want to put forward an extension to this line of reasoning, in which minimum commuting distance and excess commuting are to be seen as properties of specific spatial entities (spatially homogeneous areas) within a non-monocentric spatial system. To this end, the local minimum commuting distance is considered as a measure for spatial proximity of each relevant area, as embedded in the studied region. This measure can be used to quantify relations to other spatial characteristics such as density, spatial diversity or accessibility.

Quantification of traffic volumes is essential to assess the extent of excess travel. As a proxy for traffic volumes we will use the number of kilometres travelled per person to or from a considered zone within a pre-defined time frame. As shown in Boussauw and Witlox (2009), at least at the regional level (macro scale), distance travelled per person can be deemed a good approximation of energy consumption, sustainability of travel behaviour and total traffic volume. Depending on the spatial characteristics of the study area and the research scale level, other elements such as modal split, the composition of the fleet and the level of congestion might be incorporated to avoid running the risk of oversimplification.

In relation to Niedzielski (2006) and Yang and Ferreira (2008), the novelty of our approach is twofold. First, we develop a method that extends the use of linear programming techniques and is more adequate in studying spatial variations. In respect to the latter we are able to simulate the case where all commuters start to look simultaneously for a job closer to their homes. Second, we apply the spatially disaggregated approach at a regional scale, on an urban 
network with overlapping commuter areas. Ultimately, the methodology is applied to the study area (Flanders and Brussels) and the results are interpreted.

\section{Spatial variations in excess travel}

As a starting point, Hamilton (1982) considered a monocentric urban model, as developed by Mills (1967) and Muth (1969). Within this model, there is a balance between accessibility to the central business district (CBD) and the bid rent, which is materialized in a density gradient of both jobs and houses. The monocentric model allows predicting the minimum home-work distances on the basis of the geometric characteristics of a circle. Hamilton (1982) calculated these minimum distances for a number of American and Japanese cities. He found that these distances differed substantially from the actual commuting distances drawn from survey data of the considered cities, questioning the validity of the monocentric city model. Hamilton (1982) found an average actual commuting distance of 8.7 miles $(13.92 \mathrm{~km})$, corresponding to a minimum commuting distance of only 1.1 miles $(1.76 \mathrm{~km})$. Thus, in this case the excess rate amounts to 7.9 or, put differently, $87 \%(=(8.7-1.1) / 8.7)$ of the actual commute is excessive. Hamilton's (1982) methodology proved very controversial, and was criticized by White (1988) and Small and Song (1992), whose results showed large deviations compared to the values calculated by Hamilton. The common motivation behind these studies is the investigation of the predictive power of the monocentric urban model (later extended to polycentric and dispersed urban models; Song 1995), with respect to commuting cost minimizing behaviour.

A major dichotomy appearing among studies on excess commuting is the choice of either travel distance or travel time as a proxy for the travel cost. While travel time is an intuitively appealing proxy for travel cost, there are, from environmental policy perspectives, wellgrounded reasons to use distance as a parameter. First, the adverse external effects of travel 
are more closely related to travel distance than to travel time. Second, there is the constant nature of the personal travel time budget, which means that over time urban travellers spend a constant amount of time on their daily travel (Schafer 2000).

Among the studies in which distance is used as a parameter, extreme variations are recorded across various study areas (Ma and Banister 2006). Frost et al. (1998) found an excess commuting rate of only $18.9 \%$ for London (including all inward commuting), while the ratio found by Song (1995) amounts to $81.6 \%$ for Los Angeles. Given the lack of uniformity in the methodologies used, comparing results of different studies can hardly be justified. It was found that calculated excess commuting rates decrease as the number of constraints which are explicitly controlled increases; examples include limitations originating from two-worker households and the divide between tenants and home-owners (Kim 1995), or accounting for the expectation of future job locations (Crane 1996). Furthermore, the modifiable areal unit problem (MAUP), intrinsic to the practice of spatial analysis, adds to the difficulty of comparing the results of different studies in the sense that the size and configuration of the zones used may bias the excess commuting rate (Horner and Murray 2002).

Ma and Banister (2007) examined the theoretical relationship between variations in the spatial distribution of population and jobs, and excess commuting. They showed that the excess commuting rate is a good proxy for the potential reduction of commuting distances within a specified study area, but that the minimum commuting distances (and by extension also maximum commuting distances) are more suitable to use in comparisons between different cities or points in time. Niedzielski (2006) and Yang and Ferreira (2008), studied for the first time spatially disaggregated minimum commuting distances within an urban area.

None of the above-mentioned contributions, however, analysed empirically the spatial variations of the minimum commuting distance and excess commuting rate on a regional scale. In the monocentric city model, access to the CBD is the main determinant of the urban 
structure, while in a polycentric or dispersed model accessibility to a diverse range of job and service locations is determinant. For the detection of spatial variations of excess travel within the suburbanized historically polycentric spatial structure that characterizes many urbanized regions in Europe, the application of the monocentric city model to derive minimum commuting distances makes little to no sense.

We hypothesize that there exist important regional variations of minimum commuting distances and excess travel, for which a link with spatial characteristics (e.g., density, functional mix or proximity to major transportation infrastructure), can be found. Earlier research shows that there are important differences between cities (Charron 2007), even if it is still unclear how these values evolve through a region with multiple centres and suburban areas.

\section{Possible policy implications}

In a number of studies, such as Scott et al. (1997), Frost et al. (1998) and O'Kelly and Niedzielski (2008), the focus shifts from the explanatory nature of an urban economic model to the possible relevance for spatial policies, aimed at assessing the potential reduction of undesired external effects of the traffic, such as emissions or energy consumption. In particular, Frost et al. (1998) examined the evolution of the minimum commuting distance and excess commuting rate for several British cities, and linked this evolution with spatial developments.

For a given area, the excess commuting rate can be considered as an indicator for the extent to which the particular spatial structure of this area is able to absorb shrinkage of the total traffic volume, without incurring severe economic damage. We assume that such a reduction can be caused by external factors such as rising fuel prices, extreme congestion or dissuasive policy measures (e.g., to limit emissions; Scott et al. 1997, or congestion). Alternatively, we can also 
state that shrinkage of excess travel volumes might become part of a scenario that, in the long run, will ensue from an expected continued increase of oil prices (peak oil). In addition, minimum commuting distances are an indicator of the imbalance between the residential function (considered as the origin of travel) and other functions, such as jobs, schools, shops and recreational attraction poles (which may be regarded as destinations). The effect of encouraging or just discouraging certain new developments can thus be tested against the expected impact on minimum commuting distances. A reduction of these theoretical minimum commuting distances will eventually make an effective reduction of travelled distances under the aforementioned changing external influences much easier.

A final interesting feature of our approach is the ability to assess the impact of various autonomous development scenarios on commuter traffic. In this way, a significant growth or decline in both population and employment under the influence of external factors can lead to a better understanding of the need for investments in infrastructure or public transport.

\section{Methodology}

\subsection{Premises}

The premise of our method implies that any observed departure will be matched to the nearest observed arrival, within a pre-defined time frame (e.g. morning of a working day). For each trip purpose (e.g., work), the number of departures per zone, as well as the number of arrivals, is retained, but the observed connection between origins and destinations will be cut through with the aim of minimizing the distance between those two. To apply the methodology on a real case, we assume that an origin-destination (OD) matrix is available for the selected zones, as well as a distance matrix, providing the shortest physical network distance between each pair of zones. 
This theoretical exercise does not take into account the match between origin and destination that exists in the real world. For home-work travel, this would imply that everyone who is part of the active population can be considered suitable to perform any job. Although this theoretical assumption does not necessarily correspond to a real world situation, we have made this assumption deliberately because we want to gain an insight into the theoretically maximum reduction of travelled distances.

Apart from job qualification, there are some more possible biases that should be kept in mind. We do not consider residential self-selection (Mokhtarian and Cao 2006; Van Acker et al. 2010), the trade-off between real estate prices, accessibility and environmental quality, or the income and the composition of households (Van Ommeren 2000). Also, the presence or absence of rapid transport infrastructure, such as commuter rail, was not included in the calculation. In Section 7 we will discuss the possible impact of these simplifications for our case study. Furthermore, chained trips or detours are not simulated. As a consequence, results should be considered an underestimation. In the discussion of the case study (infra), a comparison will be made between the calculated distances travelled and those reported by survey respondents.

\subsection{Linear programming and the Monge-Kantorovich mass transportation problem}

White (1988) first suggested to define the problem of the minimum required commuting distance as a conventional Monge-Kantorovich mass transportation problem, to be solved by standard techniques of linear programming (e.g., a simplex algorithm). The definition of this type of problem is often illustrated by mines that have to supply factories. All mines together produce the ore needed by all factories. Because of differences in location, the transport cost to deliver one shipment from a mine to a factory varies across the possible pairs of mines and 
factories. The problem is solved when an OD matrix is calculated, which yields the smallest total transport cost. Today several commercial and non-commercial solvers are available, offering good approximate solutions for this global minimum cost (in our case: minimum distance) and yielding a corresponding OD matrix. A popular non-commercial solver, based on the simplex algorithm, is for example lp_solve (Berkelaar et al. 2003).

In most of the excess commuting literature, the focus is on the calculated minimum commuting distance, which is usually compared with the observed total commuting distance. In his spatially disaggregated approach Niedzielski (2006) also uses the calculated OD matrix to obtain local values for the minimum commuting distance.

However, this procedure has some disadvantages. First, there is no unique solution for the mass transportation problem (Feldmann and McCann 2002), making the resulting OD matrix dependent on the software used and, to a certain extent, on the sort order of rows and columns in the cost matrix. Second, and perhaps more problematic, is that the algorithm fills as many cells as possible with zeros, mostly assigning traffic flows from one zone to only one corresponding zone. This situation is incompatible with our approach which aims at simulating the implications of the case where all commuters start to look simultaneously for a job closer to their homes. Apart from minimizing the total travelled distance (which is equivalent to the cost in the transportation problem), it is in this case also important that the optimization process is done in a geographically balanced way, obtaining the global optimum through multiple parallel local optimization processes. Because of this additional premise, we develop our own procedure instead of using a standard solver software package. Our method results in a geographically much more 'smooth' solution, avoiding results where adjacent zones show extremely differing values. 


\subsection{Algorithm}

The algorithm developed here has been implemented to assess the extent of excess commuting. The approach adopted is as follows. First, per zone trips are made as much as possible intra-zonal. Thereafter in each run, one trip from the remaining surplus or deficit is exchanged with the nearest matching zone. The distance covered by the exchanged trip is recorded. This cycle is repeated until all departures are matched with an arrival. We give a more detailed description of the process in Figure 1.

Initially, the physical distance $d_{i j}$ over the network between any pair of zones $(i, j)$ is calculated. Each zone is represented by its centroid. Using the Dijkstra algorithm, as implemented in the Network Analyst extension of ESRI ArcGIS 9.2, all shortest paths between any pair of centroids over the network were calculated. The resulting product is a symmetrical distance matrix in which both rows and columns represent every zone of the dataset. The distance for an intrazonal trip, which is originally calculated as zero, is simulated by taking half the network distance between the centroid of the considered zone and the centroid of the nearest zone. In this way, intrazonal network distance is also taken into account, which is not always done by alternative methods such as developed in O'Kelly and Lee (2005)

The combination of the OD matrix and the distance matrix provides the total distances travelled between each pair of zones, both viewed as outbound (every zone considered as origin) as well as inbound (every zone considered as destination).

The departures and arrivals within the OD matrix are then summed, so that we obtain for each zone $i$ the total number of departures $O_{i}$ as well as the total number of arrivals $D_{i}$. The minimization process starts by equalizing the number of internal trips $I_{i}$ to the number of departures $O_{i}$ (in the case where there are fewer departures than arrivals) or to the number of arrivals $D_{i}$ (in the opposite case). This number is then multiplied by the distance of the 
simulated internal trip $H_{i}^{I}$, and stored as a basic travelled distance, both outbound $\left(H^{O}\right)$ and inbound $\left(H^{D}{ }_{i}\right)$. For the next step in the process, the smaller of those two values is subtracted from the number of departures from and arrivals at the considered zone. Trips in one of the two directions are thus reduced to zero.

For the remaining surplus (if originally there were more arrivals than departures) or deficit (in the opposite case), we question whether a deficit or otherwise a surplus exists in the nearest zone $j$. If a deficit (or surplus) can be found in zone $j$, then one trip from the surplus (or deficit) of zone $i$ is used to fill up (or to receive) a part of the deficit (or surplus) of zone $j$. The distance $d_{i j}$, which should be covered to eliminate this trip, will be added to the outgoing (or incoming) distances $\left(H^{O}{ }_{i}\right.$ or $\left.H^{D}{ }_{i}\right)$ of the considered zone as well as to the incoming (or outgoing) distances $\left(H^{D}\right.$ or $\left.H_{j}^{O}\right)$ of the nearby zone.

This process is pursued step by step until all zones $i$ are given an initial chance to exchange trips ends. Then the cycle is repeated until all surpluses and deficits are eliminated. We are particularly interested in the total minimized outbound distance $T_{i}^{O}$ and the total minimized incoming distance $T^{D}{ }_{i}$. In combination with the observed outbound distance $\left(T^{O}{ }_{i}(o b s)\right)$ and the observed inbound distance $\left(T^{D}(o b s)\right)$ for the considered zone $i$, which we previously calculated, we can also map the corresponding excess rates.

The main advantage of our algorithm is that the structure of the calculated OD matrix is not arbitrary, but that the global optimum is achieved through many cycles of local optimization. Tests show that the impact of the sort order of the rows and columns on the resulting matrix is negligible. However, there is also a disadvantage to taking this approach. In comparison with a solution generated by $l p \_s o l v e$, the total minimum commuting distance yielded by our algorithm is a bit larger (11 to $15 \%$ in our tests), making it suboptimal in a mathematical sense, although still preferable from a geographical point of view. 
for every zone $i$ characterized by $O_{i}$ departures and $D_{i}$ arrivals:

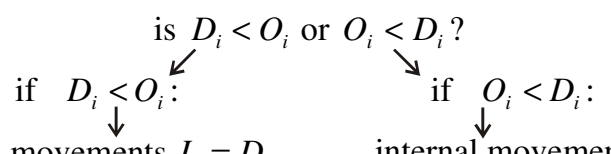

internal movements $I_{i}=D_{i}$, internal movements $I_{i}=O_{i}$, $\begin{array}{cc}D_{i}=0, O_{i}=O_{i}-I_{i} & O_{i}=0, D_{i}=D_{i}-I_{i} \\ \downarrow & \downarrow\end{array}$ external covered distance for departures $H_{i}{ }^{O}=0$, external covered distance for arrivals $H_{i}{ }^{D}=0$, $\downarrow$ select zone $j$ nearest by $i$ $\downarrow$

look up network distance $d_{i j}$ in the distance matrix $\downarrow$
calculate the internally covered distance $H_{i}^{I}=\frac{I_{i} \cdot d_{i j}}{2}$
store $H_{i}^{I}, \stackrel{D_{i}}{ }$ and $O_{i}$

select the first zone $i$ $\downarrow$

select zone $i$ that is nearest by $j$ for which:

$$
\begin{gathered}
\text { not } D_{j}=0=O_{j} \\
\text { if } D_{j}=0 \text { then } D_{i} \neq 0 \\
\text { if } \begin{array}{c}
O_{j}=0 \text { then } O_{i} \neq 0 \\
\downarrow
\end{array}
\end{gathered}
$$

look up network distance $d_{i j}$ in the distance matrix

$$
\downarrow
$$

is $D_{j}=0$ or $O_{j}=0$ ?

if $D_{j} \neq 0$ (and $\left.O_{j}=0\right)$ : $\downarrow$

calculate the externally covered distances:

$$
\begin{array}{r}
H_{i}^{O}=H_{i}^{O}+d_{i j}, \\
H_{j}^{D}=H_{j}^{D}+d_{i j}, \\
O_{i}=O_{i}-1, \\
D_{j}=D_{j}-1 \\
\searrow
\end{array}
$$

store the calculated values

$$
\text { if } \left.O_{j} \neq 0 \text { (and } D_{j}=0\right) \text { : }
$$

calculate the externally covered distances:

$$
\begin{gathered}
H_{i}^{D}=H_{i}^{D}+d_{i j}, \\
H_{j}^{O}=H_{j}^{O}+d_{i j}, \\
D_{i}=D_{i}-1, \\
O_{j}=O_{j}-1 \\
\swarrow
\end{gathered}
$$

$$
\text { is } D_{i} \stackrel{\downarrow}{=} O_{i}=0 \text { ? }
$$

if not $D_{i}=O_{i}=0$ :

select the next zone $i$ in the list

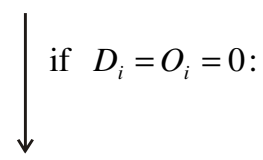

is for all zones $i: D_{i}=O_{i}=0$ ?

if not for all zones $i: D_{i}=O_{i}=0$ :

select the next zone $i$ in the list

if for all zones $i: D_{i}=O_{i}=0$ :

for all zones $i$ :

total minimized outgoing covered distance $T_{i}^{O}=H_{i}^{O}+H_{i}^{I}$ total minimized incoming covered distance $T_{i}^{D}=H_{i}^{D}+H_{i}^{I}$ 


\subsection{Excess rate}

The excess rate $E$ is defined as the ratio between the observed travelled distance $T(o b s)$ (in the model) and the calculated minimum commuting distance $T$, and is calculated both for outbound $\left(E^{O}{ }_{i}\right)$ and inbound $\left(E^{D}{ }_{i}\right)$ trips of every zone:

$$
E_{i}^{O}=\frac{T_{i}^{O}(o b s)}{T_{i}^{O}} \quad(1) \quad E_{i}^{D}=\frac{T_{i}^{D}(o b s)}{T_{i}^{D}}
$$

We calculate the excess rate $E$ for the average departure from $\left(E^{O}\right)$ or the average arrival at $\left(E^{D}\right)$ a typical zone $i$ as follows:

$$
E^{O}=\sum_{i} \frac{T_{i}^{O}(o b s)}{T_{i}^{O}} \cdot \frac{O_{i}}{\sum_{i} O_{i}} \quad \text { (3) } \quad E^{D}=\sum_{i} \frac{T_{i}^{D}(o b s)}{T_{i}^{D}} \cdot \frac{D_{i}}{\sum_{i} D_{i}}
$$

While the average excess rate over the whole study area is given by:

$$
\bar{E}^{O}=\bar{E}^{D}=\frac{\sum_{i} T_{i}^{O}(o b s)}{\sum_{i} T_{i}^{O}}=\frac{\sum_{i} T_{i}^{D}(o b s)}{\sum_{i} T_{i}^{D}}
$$

In case the data set is acquired by different sub models, as described in the 'data' section infra, a specific application of the MAUP might occur leading to a small error so that in practice $\bar{E}^{O} \neq \bar{E}^{D}$. The reason is that every sub model, of which the results will be combined into one entity, provides only detailed zoning in its own focus area (in the case of our data: a province). We overcome the aggregation error by means of the following approximation:

$$
\bar{E}=\frac{\sum_{i} T_{i}^{O}(o b s)+\sum_{i} T_{i}^{D}(o b s)}{\sum_{i} T_{i}^{O}+\sum_{i} T_{i}^{D}}
$$

We define the observed average distance covered per trip $\bar{h}(o b s)$ as follows: 


$$
\overline{h^{o}}(o b s)=\overline{h^{D}}(o b s)=\frac{\sum_{i} T_{i}^{o}(o b s)}{\sum_{i} O_{i}}=\frac{\sum_{i} T_{i}^{D}(o b s)}{\sum_{i} D_{i}}
$$

while we define the minimized average distance per trip:

$$
\overline{h^{O}}=\overline{h^{D}}=\frac{\sum_{i} T_{i}^{O}}{\sum_{i} O_{i}}=\frac{\sum_{i} T_{i}^{D}}{\sum_{i} D_{i}}
$$

Again, an error may occur by aggregating sub models, so that in practice $\overline{h^{o}}(o b s) \neq \overline{h^{D}}(o b s)$ and $\overline{h^{o}} \neq \overline{h^{D}}$. In that case, we approximate $\bar{h}(o b s)$ and $\bar{h}$ as follows:

$$
\bar{h}(o b s)=\frac{\sum_{i} T_{i}^{o}(o b s)+\sum_{i} T_{i}^{D}(o b s)}{\sum_{i} O_{i}+\sum_{i} D_{i}} \quad \text { (9) } \quad \text { and } \quad \bar{h}=\frac{\sum_{i} T_{i}^{o}+\sum_{i} T_{i}^{D}}{\sum_{i} O_{i}+\sum_{i} D_{i}}
$$

\subsection{Spatial distribution and density ratio of arrivals and departures}

The minimum commuting distance consists of a combination of both the spatial separation of functions and a difference in density between typical residential areas and typical employment centres. We will quantify these properties by defining density ratios between departures and arrivals.

We calculate the density $C$ of the departures $O$ and arrivals $D$ for the typical zone $i$ with area $A$ from which an average trip departs or at which an average trip arrives as:

$$
C^{O}=\sum_{i} \frac{O_{i}}{A_{i}} \cdot \frac{O_{i}}{\sum_{i} O_{i}} \quad(11) \quad \text { and } \quad C^{D}=\sum_{i} \frac{D_{i}}{A_{i}} \cdot \frac{D_{i}}{\sum_{i} D_{i}}
$$

While the average density over the entire study area is given by:

$$
\bar{C}^{O}=\bar{C}^{D}=\frac{\sum_{i} O_{i}}{\sum_{i} A_{i}}=\frac{\sum_{i} D_{i}}{\sum_{i} A_{i}}
$$


Spatial separation of the considered functions can thus be measured by calculating the ratio between $C^{O}$ and $C^{D}$ :

$$
R^{O D}=\frac{C^{O}}{C^{D}}
$$

\section{Case study area: Flanders and Brussels (Belgium)}

Within the context of this research we want to test our model for the Flanders and Brussels region. It is important to take into account that in this region commute areas of different cities overlap (Van Nuffel 2007), and that many jobs are located far outside the CBDs, such as in port areas, small towns, historically developed businesses outside of urban areas or peripheral industrial sites. Even though spatial dispersal is usually larger in the residential function than in other functions, a polycentric and partly dispersed spatial distribution of both jobs and other destinations should be taken into account. The polycentric nature of regional employment has a historical basis, while sprawl is mainly a post-war phenomenon that is still developing (Vandenbulcke et al. 2009). Riguelle et al. (2007) note that contemporary polycentric development, in the form of sub-centres in the periphery of major cities ('edge cities') has hardly occurred in Belgium. Moreover, Brussels, and to a lesser extent Antwerp and Ghent, are dominant employment centres, putting the importance of other historical centres into perspective (Aujean et al. 2005).

Brussels (with more than 1 million inhabitants) is the main centre of service industries and government activities and is also the largest employment centre in Belgium. The economy of Antwerp (about 500,000 inhabitants), the second largest city in Belgium and one of the largest

ports in Europe, is based on port activities and industries (e.g., petrochemicals). Ghent (about 240,000 inhabitants) is the next urban area in the ranking, with significant activity in industry, port operations and research and development. Major centres in the immediate sphere of 
influence of Brussels and Antwerp are Mechelen, Leuven, Aalst and Sint-Niklaas. In the east we find the double-centre of Hasselt-Genk, which developed around the long gone mining industry, but managed to attract new businesses. In the southwest we find the RoeselareKortrijk region characterized by smaller-scale industrial activities, while Bruges (in the northwest) is oriented towards tourism and limited port handling. The coastal area is dominated by an elongated urban network which is mainly based on tourism.

The framework for the description of spatial structures at the macro scale in this region is the so-called Spatial Structure Plan for Flanders (RSV 1997/2004). The RSV is the overarching spatial policy plan for the Flanders region. Among other issues, the RSV selects and demarcates urban areas, for which specific urban planning policies are defined.

\subsection{Data}

The methodology can be applied at different scale levels. Within the scope of this paper we focus on the level of census wards, corresponding with neighbourhoods. This unit division allows the development of a detailed analysis of the theoretical minimum commuting distances and excess traffic generation. This implies having detailed data. We use the OD matrices of the multimodal model for Flanders (MMM) (Verhetsel 1998). MMM is a macroscopic traffic simulation model that was commissioned by the Flemish government and has been developed since 1998. The model is essentially made up of five sub models, one for each province of Flanders, including the Brussels-Capital region for consistency. Every sub model consists of a GIS map that divides the province into small traffic analysis zones (TAZs). In most places, TAZs correspond to standardized census wards. To obtain homogeneous densities, some repartitioning was done. In sparsely populated areas different census wards were regrouped into one TAZ. In other places, such as the port areas, a more refined zoning was applied. The surrounding areas (the other sub modelled provinces, the 
Walloon Region, France, the Netherlands, Germany and Luxembourg) are also part of the GIS map, but are divided into TAZs of which the size increases with the distance from the study area. Properties of the used TAZs can be found in Table 1.

\begin{tabular}{|l|r|r|r|}
\hline & \multicolumn{1}{|l|}{ Total } & \multicolumn{1}{|l|}{ Mean } & \multicolumn{1}{l|}{ St. Deviation } \\
\hline Number of TAZs & 6,652 & - & - \\
\hline Area $\left(\mathrm{km}^{2}\right)$ & $13,751.36$ & 2.07 & 2.80 \\
\hline Number of departures TAZ (4-11 a.m.) & $2,356,461$ & 354 & 406 \\
\hline Number of arrivals TAZ (4-11 a.m.) & $2,356,461$ & 354 & 692 \\
\hline
\end{tabular}

Table 1. Properties of the used TAZs

The GIS map is linked to an OD matrix which indicates for a certain period of time how many trips occur from every zone to any other zone in the model. Each provincial model contains roughly between 1,400 and 3,300 zones, and the associated matrices have as many rows as columns. The matrices that were available for this study simulate traffic on an average weekday between 4 a.m. and 11 a.m. (i.e., morning traffic). By aggregating the data for a largely extended morning rush period, we avoid inaccuracies caused by the calibration of the MMM, which is designed to calculate traffic flows on an hourly basis.

The matrices were first built on data on home-work commuting and home-school commuting from the General Socio-Economic Survey 2001 (SEE 2001; Verhetsel et al. 2007), that are available at the level of census wards. SEE 2001 is an exhaustive survey of the Belgian population (excluding children younger than six years), which has its origin in the decennial census. The questionnaire of SEE 2001 gathers each individual's residence address and the address of the workplace or school. While $83.2 \%$ of the respondents provided the name of the municipality of the workplace or school, only $56.4 \%$ filled out the full address. This is significantly lower than the overall SEE 2001 response rate of 95\% (Verhetsel et al. 2007). The processed data were aggregated by neighbourhood and supply a picture of the daily travelled distances to and from each neighbourhood. This information can also be aggregated 
for analysis at the municipal level. This data was geocoded; errors and deficiencies such as the lack of addresses were corrected wherever possible. To this end, alternative socioeconomic databases were used, which were supplied by the Crossroads Bank for Enterprises ${ }^{1}$ (for home-work travel) and the Flemish Department of Education (for home-school travel). For other kinds of travel, grouped as recreation, shopping and other traffic, synthetic matrices were built using a gravity model of which the parameters were derived from the Travel Behaviour Survey in Flanders and other relevant surveys conducted in Belgium. In this way, a complete OD matrix for the base year 2007 was obtained.

Given the significant share of home-work and home-school travel during the morning peak hours, one can state that the OD matrices represent an adequate simulation of personal mobility in the morning. Nevertheless, caution is required with respect to the interpretation of the results for trip purposes other than work or school because for these trips the data is of lower accuracy. To illustrate, on the basis of the datasets used, we cannot make sufficiently accurate simulations for the traffic outside the peak hours (which consists mainly of other than home-work and home-school based trips), particularly during weekends or vacation periods.

The zoning of the MMM, which is based on census wards, was done in a more refined way for densely populated areas than for those areas that are sparsely populated. While it is logical to define zones with a homogeneous density, this has the disadvantage that the effect of the specific travel behaviour of a small population in a large but sparsely populated zone could easily result in a disproportionately prominent spot on the map. This problem can be partly alleviated by using a predetermined density threshold below which no data is represented.

Even though the MMM provides data for non-work travel too (Hammadou et al. 2008), within the framework of this paper, the calculation and discussion is done for home-work travel only. This is plausible since home-work trips are in an economic sense the most crucial of all

\footnotetext{
${ }^{1}$ The Crossroads Bank for Enterprises is the public data management service of the Belgian social security system.
} 
personal trips. This can be illustrated using price elasticities. Home-work travel in particular is a lot less price elastic, and thus more inert, than other types of trips, such as leisure or shopping travel (Mayeres 2000). An additional reason to focus on home-work travel is the fact that the availability of data about this commuting class is generally better, and that those datasets usually are more complete and reliable (Witlox 2007).

\subsection{Network}

We use Streetnet as a network to connect the various zones. Streetnet is a detailed topological representation of the Belgian road network built up by links and nodes to which various attributes, for example, road classification, are attached. To calculate the shortest networkbased path between each pair of centroids of the considered zones, we use the seven highest functional road classifications from the Streetnet network data. These categories include all regional and local roads that could be used as a connection. The two lowest categories which cover alleys and rural roads are not included. The public transport network is also not included, since we assume that the search for the shortest road between two points usually results in a shorter path than a search for the shortest link through a line of public transport.

For travel outside Belgium, where a lower accuracy is acceptable, we manually extend the Streetnet file to areas outside the country borders with a square grid with a mesh of $5 \mathrm{~km}$ in the immediate vicinity of the borders, and a mesh of $10 \mathrm{~km}$ in the more remote areas.

\section{Application and results of the case study}

From the available MMM data we deduce a data set containing only home-work travel. The calculation is done for each sub model separately, for the time frame of a weekday morning. 
The resulting maps of the five sub models are then combined into one map, covering Flanders and Brussels.

The detailed zoning of the MMM allows the detection of variations both in the observed commuting distances and the minimum commuting distances on Flanders' scale, but also allows us to make a more detailed analysis and to discover relations to spatial characteristics of neighbourhoods.

Further, it is also possible to make a typological classification (e.g., industrial area, suburban allotment, business district, nineteenth-century belt, historic town centre, ribbon development, peripheral built-up area, etc.), and then to seek explanations for variations of (minimum) commuting distances within a selected typology (e.g., distance to economic cores, distance to the main road network, supply of public transport, etc.). This form of analysis, however, falls outside the scope of this paper and will be subject to further research.

\subsection{Spatial variations of the minimum home-work distance}

Figures 2 and 3 show the calculated minimum commuting distances, based on departures and arrivals in the morning traffic. The zones with a density of departures or arrivals below the 10th percentile were omitted to avoid disproportional dominance of large but sparsely populated areas on the map.

For the entire study area the following values were found:

$$
\bar{h}(o b s)=16.2 \mathrm{~km} \quad \text { and } \quad \bar{h}=6.9 \mathrm{~km}
$$

The value for $\bar{h}(o b s)(16.2 \mathrm{~km})$ differs from the reported value of $19.0 \mathrm{~km}$ which is given by SEE 2001 (Verhetsel et al. 2007). Hence, an underestimation of the reported situation by our model is found, which is caused by chained trips and detours that were not taken into account. 


\subsubsection{Origins}

Taking Figure 2 as a reference, the following areas, considered as departure areas or origins, display low values (which we situate in the lowest distance class on the map, e.g., 0-3.20 km). This can be interpreted in a positive sense, since low values indicate a high degree of spatial proximity between home and work locations, and thus marks opportunities for short travel distances.

The metropolitan region of Brussels (1), Antwerp (2) and a wide belt between these two cities stand out. In Antwerp, the port plays an important role. Parts of the metropolitan region of Ghent (3) show rather low values. However, some rather mono-functional residential areas score relatively poorly, including some densely populated parts of the nineteenth-century belt. Like in Antwerp, in the northern part of Ghent the nearby port is decisive. The highly dispersed southwestern region of Roeselare-Kortrijk (4), the urban network of the coast (5) and the regional urban areas and small urban areas are in general in the same case. There is a wide variety of ranges of influence between the different regional urban areas. A number of specific areas show low scores as well. These are usually characterized by a rather low population density in combination with a local concentration of employment.

Following areas, among others, display high values (which we situate in the higher distance classes on the map, i.e., $>8.20 \mathrm{~km})$. The broad north-south oriented belt located between Ghent (3) and Brussels (1) stands out, with the highest values being recorded in the south (6). Also a corresponding belt located east of Brussels shows high values (7). Finally, the rather remote parts of the east-west oriented axis of the E40 highway $(8,9)$ are in the same case. The high values, obtained for these areas, can be interpreted in a negative sense, since these indicate a poor degree of spatial proximity and actual travel distances that are necessarily large. 


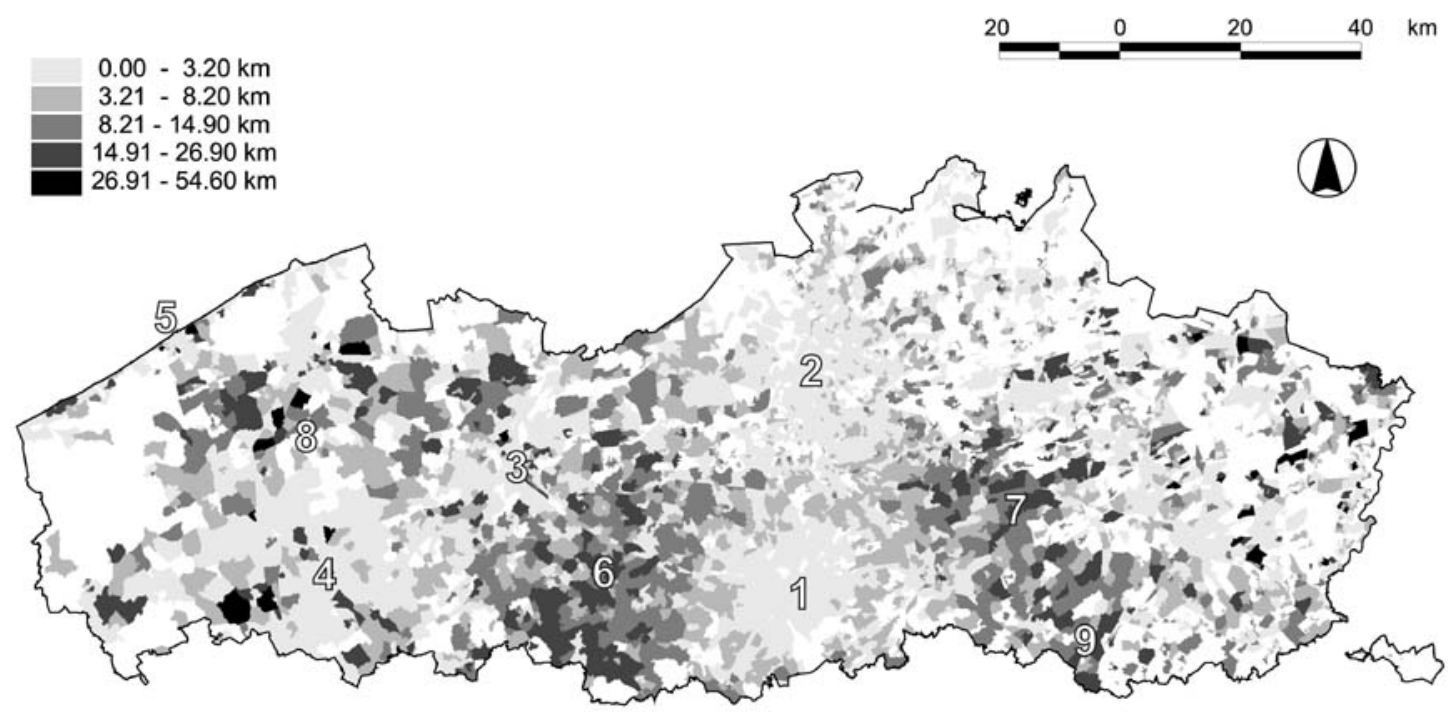

Fig. 2. Theoretical minimum commuting distance in home-work travel, origin zones during morning traffic

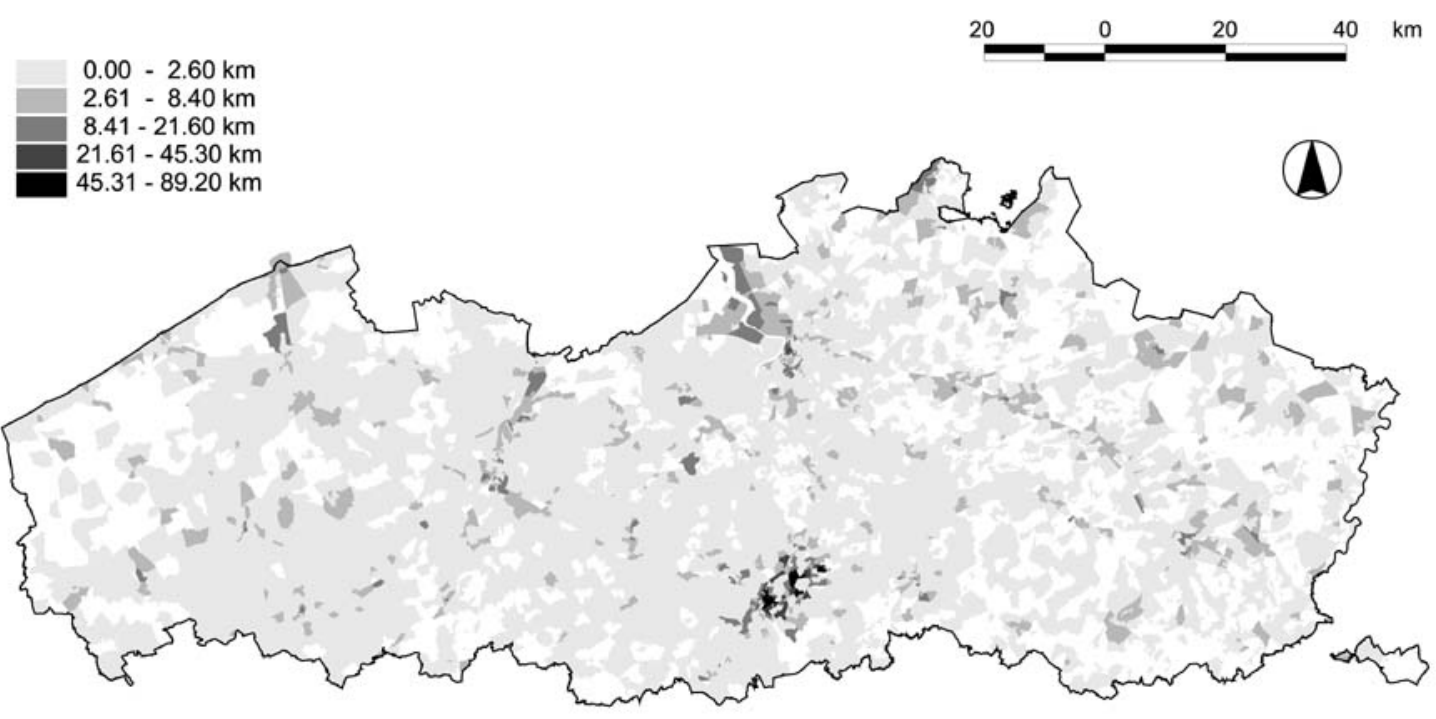

Fig. 3. Theoretical minimum commuting distance in home-work travel, destination zones during morning traffic 


\subsubsection{Destinations}

Also in the arrival zones (destinations), in this case to be considered as employment centres, there are significant differences in the distance within which employees can be found for the available jobs. From the explained perspective, low values (situated in the lowest distance class on the map) can be interpreted as positive. Typically residential areas, where the supply of active labour force is high but only few jobs are available, show low values. This is the case in most areas because jobs occur in stronger spatial concentrations than houses do. Urban neighbourhoods with a good balance between housing and jobs are in the same case.

Some specific areas display high values (situated in the higher distance classes on the map), which can be interpreted as negative. Port areas and other industrial areas with a high concentration of jobs stand out in this sense. Also parts of administrative city centres, and especially the districts in the Brussels capital region, that comprise a large share of offices, show high values.

\subsection{Spatial variations of the excess rate}

Figures 4 and 5 show the excess rate $E$ for departure zones and arrival zones. The same density thresholds were used as in Figures 2 and 3.

For the study area the following values were found:

$$
E^{O}=16.9, \quad E^{D}=16.0 \quad \text { and } \quad \bar{E}=2.33
$$

On the one hand, the high values which we find for $E^{O}$ and $E^{D}$ indicate that for a typical trip a lot of profit could be made. On the other hand, optimization of commuting in areas with a relatively high density would in the first instance have a negative impact on the areas with low density, which explains the rather low figure we find for the global excess rate $\bar{E}(=2.33$, or, put differently, $57 \%$ of the actual commute is excessive). Despite the proportionally large 
profits which could be obtained in high density areas, a minimization of the commuting distances would lead to an overall reduction of only a factor of 0.43 .

\subsubsection{Origins}

Areas with a high excess rate are typically located in urban areas or, more specifically, near major concentrations of employment. That is because in these regions the minimum commuting distances are very small. At the same time the accessibility of jobs is usually higher than in the more remote areas, so that the physical distance criterion will be less preponderant in job choice.

These findings are in line with what Hamilton (1982) has found, namely, that city-dwellers go to work many times further from home than is suggested by the spatial distribution of housing and jobs. The explanation is found, on the one hand, in the theory of the constant travel time budget and, on the other hand, in the financial travel budget being a constant share of the household income (Schafer 2000). Departing from an urban area, there are a lot more jobs available within reach of the available generalized personal travel budget than departing from a more rural area. Viewed from the urban area, the job that yields the greatest benefit will often not be the nearest job, compared to the viewpoint from the countryside.

Nevertheless, net commuting distances departing from the urban areas are still shorter than average. In summary, the spatial structure of urban areas ensures that the commuting distances are relatively low, but that there still exists a wide margin that allows an additional reduction of travelled distances.

Note that some more suburban regions can be found that have both a high excess rate and long commuting distances. Those are generally areas that are easily accessible and have high incomes so that the barrier to travel over long distances is far lower there. 


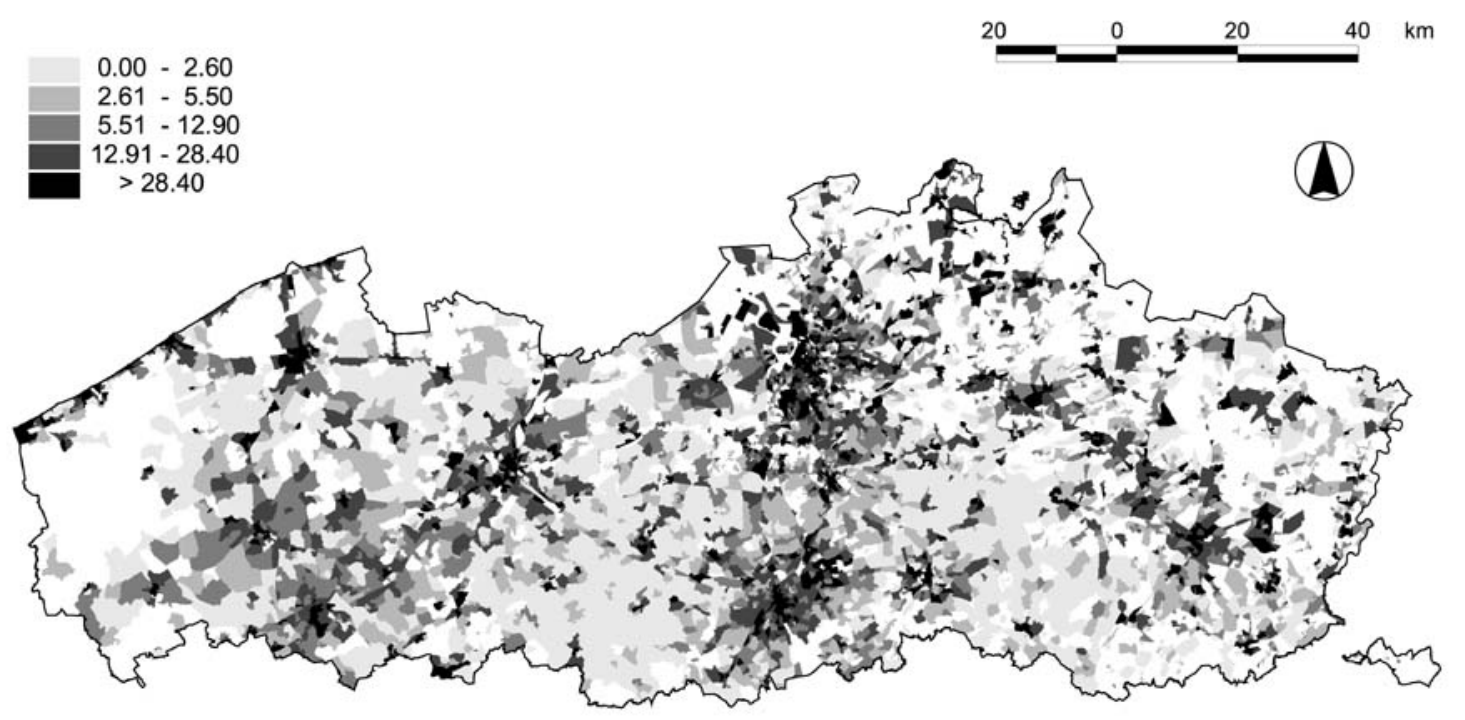

Fig. 4. Excess rate in home-work travel, origin zones during morning traffic

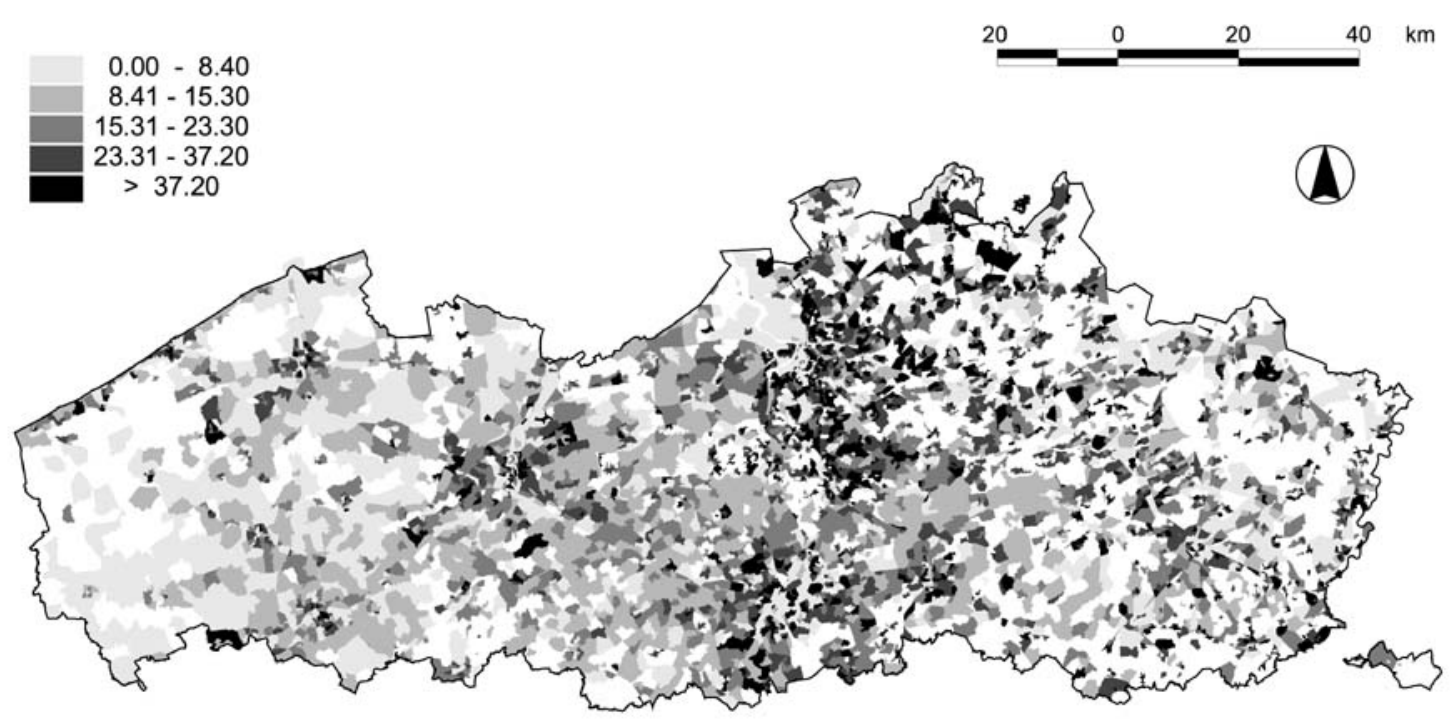

Fig. 5. Excess rate in home-work travel, destination zones during morning traffic 
As opposed to these urban and suburban areas are the more remote municipalities, mostly belonging to the rural areas. Most of these municipalities have an excess rate of around 1, or often even lower than 1. Again, these low values could be explained by the high excess rate in the core municipalities. Many workers who live in those core municipalities still go to work far from home, and thus make the nearby jobs available for residents of the surrounding municipalities. The very high excess rates in the core municipalities are responsible for the counterintuitively low excess rate in the surrounding municipalities.

The municipalities with a very low excess rate are the most vulnerable to changes in external factors that steer travel behaviour. When the generalized cost of trips would increase (e.g., by rising fuel prices, congestion, road pricing or deterioration of the supply of public transport), employees will be inclined to look for a job closer to home. At the time that residents of core municipalities are going to work closer to home, this would mean that the residents of the surrounding municipalities with a low excess rate would have to go to work even further from home.

\subsubsection{Destinations}

Zones with a low excess rate are typically found in areas with high concentrations of employment, such as the port areas and other large-scale industrial cores and city centres. In the case of large-scale industry, this is somewhat remarkable because in most cases the observed commuting distances to such locations are already considerably higher than average. The physical separation of functions plays here: for the industries that are established at these, often remote, locations it appears difficult to attract employees from a small recruitment area. A similar phenomenon as in the large industrial cores occurs in the office centres in Brussels. Also in the region Roeselare-Kortrijk we notice low values, which are in this case, however, linked to short observed commuting distances. In this region the actual travelled distances are 
more often than average approaching the optimum. In the north-south oriented belt between Ghent and Brussels, we notice again low values, but given the limited employment there, this should not be evaluated positively.

High excess rates are typically found in both sparsely and densely populated residential areas, where the relatively limited number of available jobs is often occupied by employees who do not necessarily live in the vicinity.

\subsection{Spatial distribution and density ratio of arrivals and departures}

The density of a zone from which an average trip departs $C^{O}$ is 1,187 departures $/ \mathrm{km}^{2}$, while for the average arrival $C^{D}$ is 4,756 arrivals $/ \mathrm{km}^{2}$. The ratio between the two density measures

$R^{O D}=0.25$. The average density of arrivals and departures $\bar{C}^{O}=\bar{C}^{D}=171$ trips $/ \mathrm{km}^{2}$.

The large average disparity in concentrations of jobs and houses sheds light on the spatial background of the minimum commute distance. In the specific case of home-work travel, this ratio is of course strongly related to the more commonly used jobs-housing balance parameter (Peng, 1997).

\section{Possible biases}

In the context of Flanders and Brussels, the mentioned possible biases, originating from our premises, will be more serious when we consider the larger cities, particularly Brussels. In Belgium, highly specialized, well-paid jobs are mostly centralized in the Brussels region, where the CBD plays an important role. The long distance rail accessibility to this $\mathrm{CBD}$ is excellent, while the geographically central location in the Brussels agglomeration ensures the interaction with a large number of potential employees who live in the surroundings. These factors result in specialist workers in Brussels not living in the city. Instead, they prefer the 
green suburban neighbourhoods in Brussels' periphery, or in the less densely built municipalities of the large commuting region. The spatial mismatch between locations of work and residence of specialized employees is an additional obstacle to a possible reduction of commuting distances, occurring particularly in the large cities, and especially in Brussels. A similar bias is found in the spatial variation of household sizes. One-person households, which are encountered more often in cities, face more freedom regarding the choice of job and residence location than families with two breadwinners.

\section{Conclusions}

In this paper, we have studied the spatial variation of the minimum commuting distance and the excess rate as indicators of spatial proximity of functions, in particular housing and jobs. We have elaborated a methodology to calculate these indicators, and indicated their relevance for spatial planning policies. Methodological problems associated with the use of those indicators still occur, especially when different regions or cities are compared with each other. However, the spatial variation of the minimum commuting distance seems suitable to measure the extent to which a given area can operate on the basis of short distances. The main reason for these variations is the systematic differences in the spatial distribution of the job market with respect to the housing market.

In Flanders and Brussels jobs, but also services, show a much stronger pattern of concentration than dwellings do. Moreover, suburbanization of both functional groups happens in a different way. Employment is mainly situated in or in the immediate sphere of influence of urban areas. Furthermore, extremely high local concentrations of employment exist, such as in the Brussels office districts, the seaports or the national airport. Employers who are located in these areas are usually unable to recruit workers living on average close to the company. 
The suburbanization of dwellings is for a major part located in municipalities in the countryside, often far away from the economic core areas. Although we find the highest concentrations of this residential function in the urban areas, housing is much more homogeneously spread over the entire study area than jobs. This means that for inhabitants of the more remote regions with a low jobs-housing ratio it is very difficult to find a job close to home.

The minimum commuting distance can be considered as a measure of proximity to the labour market, viewed from the housing market, or vice versa, as a measure of proximity to the housing market, viewed from the labour market. The model that is discussed in this paper suggests that the minimum average distance for a home-work trip within the current job and housing market in Flanders and Brussels is fixed at $6.9 \mathrm{~km}$, to be compared to a calculated real world value of $16.2 \mathrm{~km}$. However, in this variable important regional gradients can be observed. Employees living in the vicinity of the economic core areas can easily find a job close to home, whereas the inhabitants of remote rural municipalities must necessarily commute over long distances.

The regional variations of the excess rate show that people who live in the vicinity of major employment centres could still significantly reduce their daily commuting distance, relatively spoken. However, for residents of outlying regions this would be difficult or even impossible: they will instead be required to travel even longer distances in case a general contraction of commuting distances would happen. Such a reduction of commuting distances is a scenario that may occur when the absolute cost of transportation would increase.

To date, in the Western world we have only observed a trend of increasing commuting distances, an evolution originating in the democratization of the car. Since the fastest way to make a typical journey to work is by car, this has led to higher speeds and ultimately to trips covering increasingly longer distances. 
The spatial segregation of functions in Flanders and Brussels mainly developed in the era of upcoming cheap, fast transportation. We can therefore say that the longer distances travelled partly materialized in the form of the physical separation of functions. To a certain extent, this spatial development shuts the door to a potential shrinkage of commuting distances. Regions with a large minimum commuting distance are therefore very sensitive to rising transport costs, leading to a reduction of mobility. In the long run, however, such an increase in costs is likely to occur in the light of peak oil.

Although the paper only deals with home-work commuting, a similar logic may be valid for services such as schools and shops. Spatial proximity of functions is a paramount prerequisite for a sustainable travel pattern on the basis of relatively short distances. Towards policy making, this can be translated as the importance of providing an adequate spatial and functional mix. Given the relatively large average daily distance that is covered per person, the role of this spatial mix is probably more important at a regional level than at the level of, for example, a historical urban structure (compact city). Concretely, this could mean that the stimulation of additional jobs and services in areas with a low jobs-housing balance should be given priority, but also that suburbanization of the residential function in remote rural municipalities should be discouraged.

Mapping the development of minimum commuting distances is an important research field. For a better understanding of the evolution of this indicator, it is necessary to compare longitudinal data. Further research should also include non-work-related trips, and should take into account other biases, such as the influence of accessibility (in terms of travel time), the role of chained trips, household composition and income, and modal choice. 


\section{References}

Aguilera A (2005) Growth in commuting distances in French polycentric metropolitan areas: Paris, Lyon and Marseille. Urban Studies 42: 1537-1547

Aujean L, Castiau E, Roelandts M, Vandermotten C (2005) Central Belgium: Commuting and the definition of functional urban regions. Institute of Community Studies/The Young Foundation \& Polynet Partners, London

Banister D, Watson S, Wood C (1997) Sustainable cities, transport, energy and urban form. Environment and Planning B 24: 125-143

Berkelaar M, Eikland K, Notebaert P (2003) lp_solve, version 4.0. http://groups.yahoo.com/group/lp_solve/

Boussauw K, Witlox F (2009) Introducing a commute-energy performance index for Flanders. Transportation Research Part A 43: 580-591

Charron M (2007) From excess commuting to commuting possibilities: More extension to the concept of excess commuting. Environment and Planning A 39: 1238-1254

Crane R (1996) The influence of uncertain job location on urban form and the journey to work. Journal of Urban Economics 39: 342-356

Feldman M, McCann RJ (2002) Uniqueness and transport density in Monge's mass transportation problem. Calculus of Variations and Partial Differential Equations 15: 81-113

Frost M, Linneker B, Spence N (1998) Excess or wasteful commuting in a selection of British cities. Transportation Research Part A 32: 529-538

Giuliano G, Small KA (1993) Is the journey to work explained by urban structure? Urban Studies 30: 1485-1500

Hamilton BW (1982) Wasteful commuting. The Journal of Political Economy 90: 1035-1053 
Hammadou H, Thomas I, Verhetsel A, Witlox F (2008) How to incorporate the spatial dimension in destination choice models: The case of Antwerp. Transportation Planning and Technology 31: 153-181

Horner MW, Murray AT (2002) Excess commuting and the modifiable areal unit problem. Urban Studies 39: $131-139$

Horner MW, O'Kelly ME (2007) Is non-work travel excessive? Journal of Transport Geography 15: 411-416

Kim S (1995) Excess commuting for two-worker households in the Los Angeles metropolitan area. Journal of Urban Economics 38: 166-182

Ma KR, Banister D (2006) Excess commuting: A critical review. Transport Reviews 26: 749-767

Ma KR, Banister D (2007) Urban spatial change and excess commuting. Environment and Planning A 39: 630646

Mayeres I (2000) The efficiency effects of transport policies in the presence of externalities and distortionary taxes. Journal of Transport Economics and Policy 34: 233-260

Mills ES (1967) An aggregative model of resource allocation in a metropolitan area. American Economic Review 57: $197-210$

Mokhtarian P, Cao X (2006) Examining the impacts of residential self-selection on travel behavior: A focus on methodologies. Transportation Research B 42: 204-228

Muth RF (1969) Cities and housing: The spatial pattern of urban residential land use. University of Chicago Press, Chicago, IL 
Niedzielski MA (2006) A spatially disaggregated approach to commuting efficiency. Urban Studies 43: $2485-$ 2502

O'Kelly M, Lee W (2005) Disaggregate journey-to-work data: Implications for excess commuting and jobshousing balance. Environment and Planning A 37: 2233-2252

O’Kelly M, Niedzielski MA (2008) Efficient spatial interaction: Attainable reductions in metropolitan average trip length. Journal of Transport Geography 16: 313-323

Peng ZR (1997) The jobs-housing balance and urban commuting. Urban Studies 34: 1215-1235

Riguelle F, Thomas I, Verhetsel A (2007) Measuring urban polycentrism: A European case study and its implications. Journal of Economic Geography 7: 193-215

RSV (1997/2004) Ruimtelijk structuurplan Vlaanderen - gecoördineerde versie. Ministry of the Flemish Community, Brussels

Schafer A (2000) Regularities in travel demand: An international perspective. Journal of Transportation and Statistics 3: 1-31

Scott DM, Kanaroglou PS, Anderson WP (1997) Impacts of commuting efficiency on congestion and emissions: Case of the Hamilton CMA, Canada. Transportation Research Part D 2: 245-257

Small AK, Song S (1992) Wasteful commuting: A resolution. Journal of Political Economy 100: 888-898

Song S (1995) Does generalizing density functions better explain urban commuting? Some evidence from the Los Angeles region. Applied Economics Letters 2: 148-150

Van Acker V, Van Wee B, Witlox F (2010) When transport geography meets social psychology: Toward a conceptual model of travel behaviour. Transport Reviews 2: 219-240 
Van Acker V, Witlox F, Van Wee B (2007) The effects of the land use system on travel behavior: A structural equation modeling approach. Transportation Planning and Technology 30: 331-353

Vandenbulcke G, Steenberghen T, Thomas I (2009) Mapping accessibility in Belgium: A tool for land-use and transport planning? Journal of Transport Geography 17: 39-53

Van Nuffel N (2007) Determination of the number of significant flows in origin-destination specific analysis:

The case of commuting in Flanders. Regional Studies 41: 509-524

Van Ommeren J (2000) Job and residential search behaviour of two-earner households. Papers in Regional Science 79: 375-391

Verhetsel A (1998) The impact of spatial versus economic measures in an urban transportation plan. Computers, Environment and Urban Systems 22: 541-555

Verhetsel A, Thomas I, Van Hecke E, Beelen M (2007) Pendel in België. Deel I: De woon-werkverplaatsingen. Federal Public Service Economy, SMEs, independent Professions and Energy, Brussels

White MJ (1988) Urban commuting journeys are not 'wasteful'. Journal of Political Economy 96: 1097-1110

Witlox F (2007) Evaluating the reliability of reported distance data in urban travel behaviour analysis. Journal of Transport Geography 15: 172-183

Yang J, Ferreira J (2008) Choices versus choice sets: A commuting spectrum method for representing jobhousing possibilities. Environment and Planning B 35: 364-378 